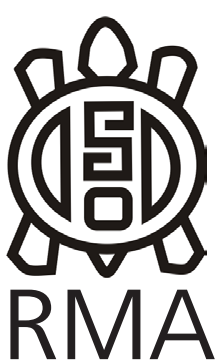

Arqueología

\title{
El sitio Abrigo Pozo Cavado, Puna de Salta. Nuevo aporte arqueológico y cronológico para el estudio de procesos de cambio hacia finales del Holoceno medio (ca. 5600-4600 años AP)
}

\author{
The Abrigo Pozo Cavado site, Puna of Salta. New \\ archaeological and chronological contributions to the \\ study of processes of change from the end of the Middle \\ Holocene (ca. 5600-4600 years BP)
}

Gabriel E. J. López* y Juan P. Orsi**

*CONICET- Instituto de Arqueología, FFyL, UBA. E-mail: gabelope@yahoo.com **Instituto de Arqueología, FFyL, UBA. E-mail: juanpabloorsi@gmail.com

\begin{abstract}
Resumen
Este trabajo analiza y discute nueva información arqueológica y cronológica del sitio Abrigo Pozo Cavado correspondiente a finales del Holoceno medio (ca. 5600-4600 años AP). Este sitio se ubica en el Salar de Pocitos, Puna de Salta, a una altura de $3700 \mathrm{msnm}$. El registro arqueofaunístico indica una predominancia absoluta de los Camelidae pero con variabilidad osteométrica relacionada con la presencia de distintas especies de esta familia. El material lítico registra distintas morfologías de puntas de proyectil y materias primas, entre ellas, obsidianas alóctonas. Por último, se destaca la presencia de cuentas de valva.
\end{abstract}

Palabras clave: Holoceno medio; procesos de cambio; Puna de Salta.

\begin{abstract}
This paper analyzes and discusses new archaeological and chronological data at the end of the Middle Holocene (ca. 5600-4600 years BP) from the Abrigo Pozo Cavado site. This site is located in the Salar de Pocitos, Puna of Salta, at an altitude of 3700 masl. The archaeofaunal record presents an absolute predominance of Camelidae, yet variability in osteometric data indicates different species of this family. The lithic material has diverse morphologies of projectile points and raw materials, including allochthonous obsidian. Finally, the presence of shell beads is also reported.
\end{abstract}

Keywords: Middle Holocene; processes of change; Puna of Salta.

Este trabajo presenta nueva información arqueológica y cronológica procedente del sitio Abrigo Pozo Cavado, ubicado en la Puna de Salta, con ocupaciones humanas en el Holoceno medio y tardío. La evidencia arqueológica procedente de este sitio aporta al conocimiento de los procesos de cambio ocurridos a partir del Holoceno medio en la Puna.

Más específicamente, se analiza y discute el registro en capa fechado entre ca. 5600 AP y ca. 4600 AP, cronología en la cual se han planteado cambios relacionados con procesos de intensificación y domesticación de camélidos (ver Cartajena et al. 2007; Olivera 1997; Yacobaccio 2001; entre otros). Estos procesos se relacionaron también con cambios en la demografía, en la movilidad, en el tamaño de los grupos y en la tecnología, entre otros aspectos (Aschero 1994; López 2008; López y Restifo 2012; Muscio 2012; Yacobaccio 2001).

El sitio Abrigo Pozo Cavado (APC) se ubica en la cuenca de Pocitos, Puna de Salta (Figura 1). Se trata de un abrigo rocoso de aproximadamente $30 \mathrm{~m}$ en línea recta, localizado en el borde del Salar de Pocitos a una altura de 3700 msnm (López 2013). La cuenca de Pocitos comenzó a ser investigada sistemáticamente a partir del año 2009, mientras que este sitio fue registrado en el año 2010 (López et al. 2013). La metodología de análisis siguió un criterio distribucional y se realizaron prospecciones y sondeos a lo largo de la cuenca. En el 


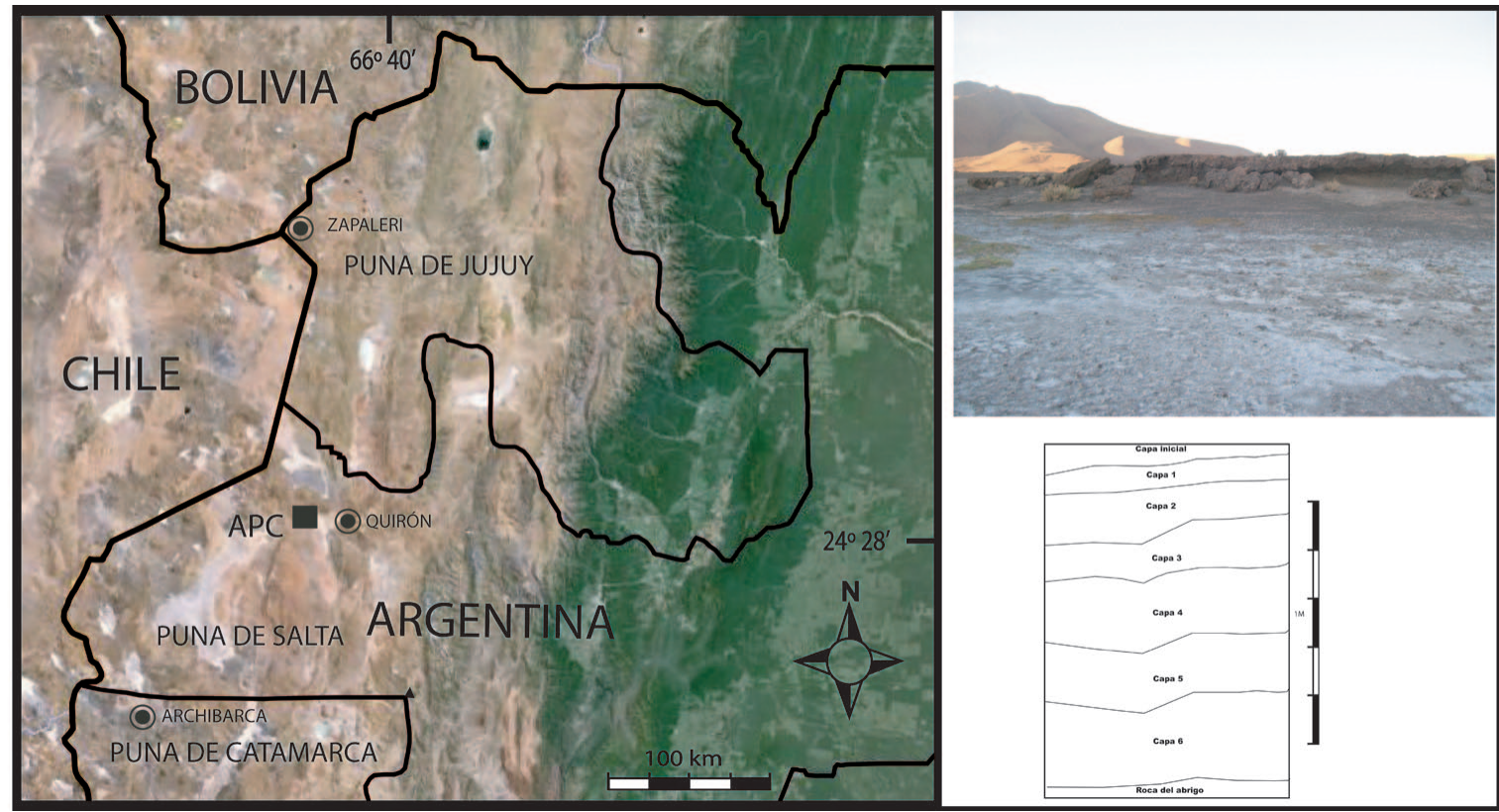

Figura 1: Mapa con la ubicación de Abrigo Pozo Cavado y las fuentes de obsidiana, y detalle del sitio y la estratigrafía.

Figure 1: Map showing the location of Abrigo Pozo Cavado and sources of obsidian, and site detail and stratigraphy.

sitio APC se realizaron hasta el momento 4 cuadrículas de $1 \times 1 \mathrm{~m}$ para testear la estratigrafía en distintos sectores. La excavación permitió registrar una amplia secuencia arqueológica (Figura 1). Las primeras capas, aunque sin fechado, corresponderían a contextos tardíos del Holoceno, con evidencia de cerámica. Las capas más tempranas presentan fechados entre el Holoceno medio y comienzos del Holoceno tardío (López et al. 2013). La estratigrafía se encuentra más completa hacia el sector sur del sitio, donde la sedimentación es mayor. Allí se realizaron las dos primeras cuadrículas (1 y 2), mientras que las otras se establecieron hacia el centro (cuadrícula 4) y en el sector norte (cuadrícula 3). En estos últimos sectores, no se registraron las capas iniciales más tardías.

La estratigrafía se compone de capas limosas y arcillosas, algunas con alta cantidad de material orgánico y carbonoso, con fechas entre 6280 años AP y 2970 años AP (López et al. 2013). En este trabajo se presentan tres nuevas dataciones procedentes de la capa 5: $5640 \pm 100$ años AP, $5480 \pm 100$ años AP, y $4620 \pm 110$ años AP (Tabla 1). Esta capa limo-arcillosa se registra en las cuadrículas del centro y el sur del abrigo, y presenta espesores variables entre 40 y $20 \mathrm{~cm}$. En la excavación no se pudieron distinguir variaciones estratigráficas dentro de la misma. Por esta razón, los conjuntos analizados aquí se consideran como parte de un componente cronológicamente extenso. En el futuro, se esperan realizar nuevos fechados de esta capa en las distintas cuadrículas con el objetivo de ajustar la cronología radiocarbónica. De todas formas, los resultados presentados permiten avanzar en las investigaciones relacionadas con los cambios ocurridos en las ocupaciones humanas a partir del Holoceno medio en el sitio. A continuación se analiza y discute brevemente la evidencia arqueológica (especialmente fauna y lítico) procedente de las cuadrículas 1, 2 y 4 de esta capa.

\section{Material arqueofaunístico}

Los conjuntos arqueofaunísticos de APC muestran una representación absolutamente predominante de camélidos al nivel de Familia. En la capa 5 los camélidos comprenden el $100 \%$ de la muestra $(\mathrm{N}=227)$. Las partes

Tabla 1: Fechas radiocarbónicas del sitio Abrigo Pozo Cavado.

Table 1: Radiocarbon dates of the Abrigo Pozo Cavado site.

\begin{tabular}{cccc}
\hline Sitio & Laboratorio & $\begin{array}{c}\text { Fecha C } \\
\text { años AP }\end{array}$ & Muestra \\
\hline $\begin{array}{c}\text { Abrigo Pozo } \\
\text { Cavado } \\
\text { Cuadrícula 1 } \\
\text { Capa 6 } \\
\text { Abrigo Pozo } \\
\quad \text { Lavado } \\
\text { Cuadrícula 4 } \\
\text { capa 5 }\end{array}$ LP-2905 & $6280 \pm 90$ & Óseo \\
$\begin{array}{c}\text { Abrigo Pozo } \\
\text { Cavado }\end{array}$ & LP-3017 & $5480 \pm 100$ & Carbón \\
$\begin{array}{c}\text { Cuadrícula 4 } \\
\text { Capa 5 }\end{array}$ & & & \\
$\begin{array}{c}\text { Abrigo Pozo } \\
\text { Cavado }\end{array}$ & LP- 3022 & $4620 \pm 110$ & Carbón \\
$\begin{array}{c}\text { Cuadrícula 4 } \\
\text { Capa 5 }\end{array}$ & & & \\
$\begin{array}{c}\text { Abrigo Pozo } \\
\text { Cavado APC/ } \\
\text { Cuadrícula 1 } \\
\text { Capa 4 }\end{array}$ & AA90384 & $3884 \pm 59$ & Óseo \\
$\begin{array}{c}\text { Abrigo Pozo } \\
\text { Cavado } \\
\text { Cuadrícula 1 } \\
\text { Capa 3 }\end{array}$ & LP- 2636 & $2970 \pm 80$ & Óseo \\
\hline
\end{tabular}




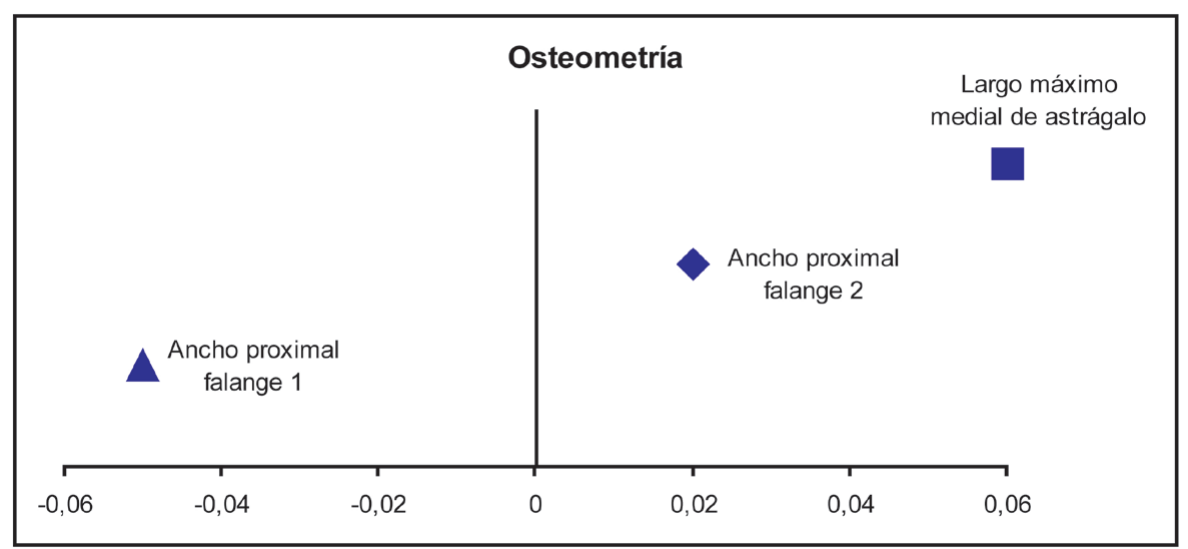

Figura 2: Osteometría de especímenes de camélidos de Abrigo Pozo Cavado en la capa de ca. 5600-4600 años AP. Diferencia de logaritmos de muestras arqueológicas en relación con el estándar actual de guanaco.

Figure 2: Osteometry of camelid specimens from Abrigo Pozo Cavado site from the layer ca. 5600-4600 years BP. Difference of logarithms of the archaeological samples in relation to the current standard of guanaco.

esqueletarias muestran una representación mayor del esqueleto apendicular (54,6 \%) sobre el axial (45,4\%) en NISP. Entre los huesos apendiculares predominan los metapodios y las falanges, mientras que en el esqueleto axial los huesos más representados son el cráneo y los dientes fragmentados, las vértebras lumbares y cervicales, y las costillas.

Otro indicador importante para abordar la problemática relacionada con la diversidad taxonómica hacia finales del Holoceno es la osteometría. Se hace referencia específicamente a la representación de distintas especies de camélidos y la posible presencia de camélidos domesticados y/o en proceso de domesticación, tal como se registró para esta cronología en otros sitios de los Andes (Mengoni Goñalons y Yacobaccio 2006). Hasta el momento, los especímenes con posibilidades de medición son escasos $(\mathrm{N}=3)$, pero constituyen un aporte al estudio de esta problemática. En especial, porque se registró un espécimen de astrágalo de tamaño similar o mayor al de las llamas actuales y más grande que el guanaco actual tomado como muestra (Mengoni Goñalons y Elkin com. pers.). Esta contribución toma más relevancia considerando la escasa cantidad de especímenes medidos para este contexto cronológico a nivel macrorregional (ver Mengoni Goñalons y Yacobaccio 2006). Para profundizar la diferencia en estos valores en relación con el estándar de guanaco, se realizaron los análisis de logaritmos de Meadow (1987) entre los especímenes actuales y arqueológicos (Figura 2). Los resultados mostraron tres tamaños distintos: uno bastante mayor que el guanaco, otro intermedio aunque mayor que el guanaco, y otro más pequeño que correspondería a vicuña. La presencia de un espécimen de tamaño grande en esta cronología podría indicar la representación de un camélido domesticado y/o en proceso de domesticación, aunque es necesario aumentar la muestra para abordar el análisis de la variabilidad.

\section{Material lítico}

Dentro del conjunto lítico analizado ( $N=129)$, se distinguieron instrumentos y desechos de talla (Figura 3). Siguiendo las categorías de Aschero (1983), se establecieron las siguientes clases de instrumentos: artefacto de formatización sumaria $(\mathrm{N}=5)$, punta de proyectil $(\mathrm{N}=4)$, fragmento no diferenciado de artefacto formatizado $(\mathrm{N}=3)$, cuchillo de filo retocado $(\mathrm{N}=1)$ y raspador $(\mathrm{N}=1)$. En general se suele plantear que las puntas de proyectil son artefactos tiempo-sensitivos (Jones y Beck 1992), y en este caso se registraron morfologías comunes en distintos contextos de los Andes (López y Restifo 2014). Se hace referencia a puntas lanceoladas mediano-grandes y pequeñas apedunculadas, y de morfología triangular o subtriangular de limbo alargado y apedunculada (Figura 4 A). Las puntas lanceoladas son recurrentes entre el Holoceno medio y comienzos del Holoceno tardío, lo cual es coherente con las fechas registradas para esta capa de APC. Por su parte, la punta triangular tiene características similares a otra hallada en el mismo sitio en la capa de
Figura 3: Porcentaje de materias primas en material lítico en la capa de ca. 5600-4600 años AP del sitio Abrigo Pozo Cavado.

Figure 3: Percentage of lithic material in the layer dated ca. 56004600 years BP of the Abrigo Pozo Cavado site.

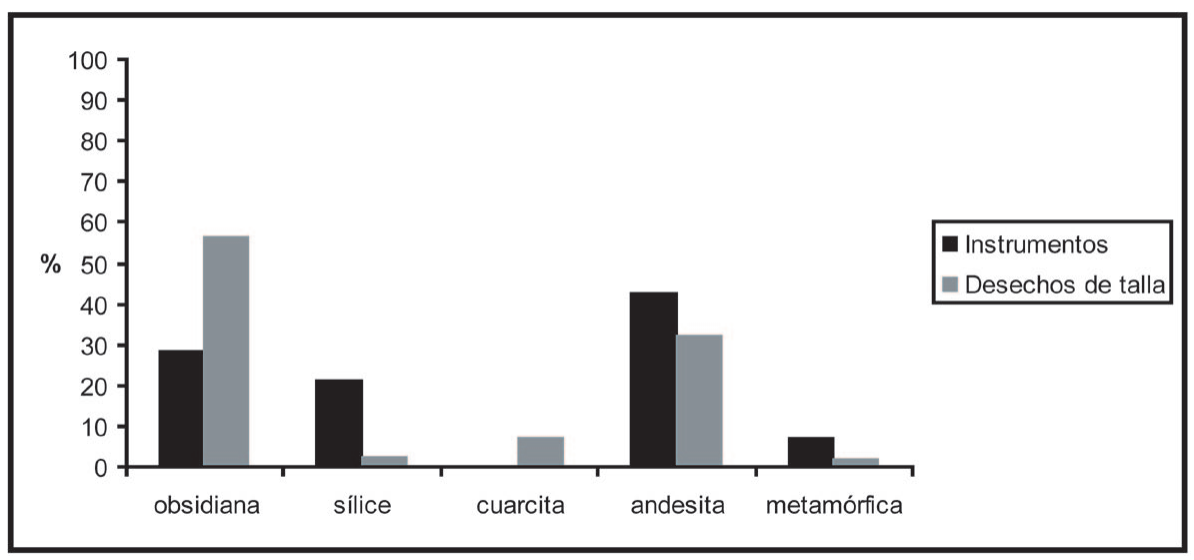


Figura 4: A. Distintas morfologías de puntas recuperadas en el sitio Abrigo Pozo Cavado (ca. 5600-4600 años AP). B. Cuentas recuperadas en el sitio Abrigo Pozo Cavado (ca. 5600-4600 años AP).

Figure 4: A. Different projectile point morphologies recovered at the Abrigo Pozo Cavado site (ca. $5600-4600$ years BP). B. Beads recovered at Pozo Cavado site (ca. $5600-4600$ years $B P$ ).

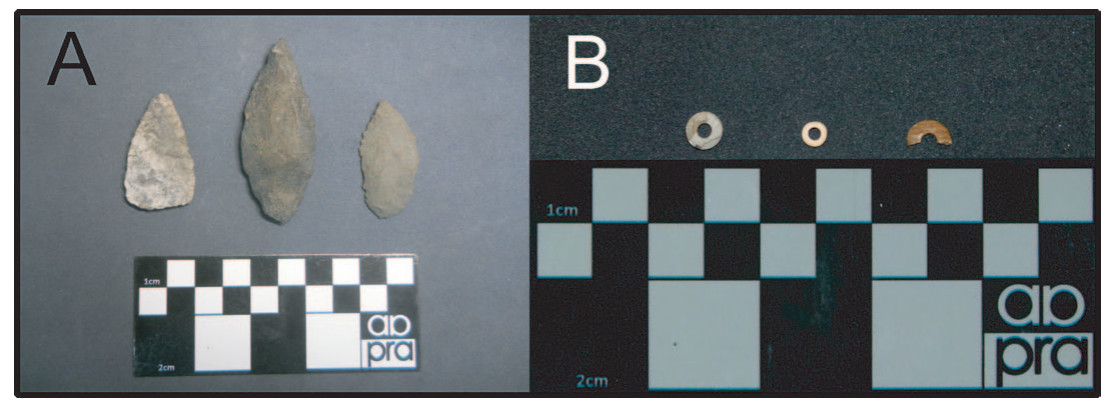

6280 años AP (López et al. 2013). De todas formas, las morfologías triangulares apedunculadas están presentes en los registros andinos desde comienzos del Holoceno temprano (Aldenderfer 1998; Núñez et al. 2005).

Los desechos de talla incluyen distintas clases de lascas, predominando las indeterminadas $(\mathrm{N}=56)$ y las angulares $(\mathrm{N}=54)$, y en menor medida las planas $(\mathrm{N}=2)$ y las secundarias $(\mathrm{N}=3)$. Esto coincide con la forma base de los instrumentos, que también corresponde a lascas. En relación con la representación de materias primas predominan las obsidianas y las andesitas (ver Figura 3). Se ha determinado la presencia mayoritaria de obsidianas de la fuente de Quirón, ubicada a unos $20 \mathrm{Km}$ de APC. Asimismo, la otra obsidiana más representada proviene de la fuente de Archibarca, distante a unos $140 \mathrm{Km}$ al sur de APC. También se detectó la presencia de un espécimen de obsidiana procedente de la fuente de Zapaleri, a unos $170 \mathrm{Km}$ hacia el norte. La determinación de las fuentes se realizó a partir del análisis de fluorescencia de rayos $X$ de diversos sitios arqueológicos de la Puna de Salta (Dr. Michael Glascock), y por comparación macroscópica. Por su parte, las andesitas son recurrentes en las puntas de proyectil lanceoladas y en los desechos de talla. Esta materia prima podría provenir de la fuente registrada en Pastos Grandes a unos $60 \mathrm{Km}$ al este de Pocitos, en el sitio Picadero (López 2008), aunque son necesarias nuevas prospecciones para determinar que no haya una fuente más cercana. En menor medida, también se encuentran representadas silíceas, cuarcitas y metamórficas (Figura 3), posiblemente de procedencia local, aunque por el momento las fuentes no fueron determinadas.

\section{Otras líneas de evidencia: presencia de cuentas}

En la capa de APC de fines del Holoceno medio se han recuperado pequeñas cuentas de valva. Se trata de un espécimen fragmentado y dos enteros, que habrían sido usados como adorno (Figura 4 B). Constituyen elementos circulares con un orificio central. Las medidas del diámetro de las cuentas son de $0,6 \mathrm{~cm}$ aproximadamente. Por el momento no se procedió a la identificación taxonómica específica. Sin embargo, en el sitio Alero Cuevas, distante $60 \mathrm{~km}$ de APC, se recuperaron cuentas de un bivalvo marino del Océano Pacífico (Clamys sp.) y dos de agua dulce (Anodontites o Diplodon) (López 2008). Asimismo, en la capa de 3884 años AP de APC se recuperaron otras dos cuentas de valva enteras, mientras que en el sitio Abrigo Bella Vista, también en Pocitos, se registraron más hallazgos de estas características. Este último sitio por el momento no presenta fechas radiocarbónicas.

\section{Discusión y conclusiones}

Hacia finales del Holoceno medio se han detectado indicadores de cambios a nivel macrorregional relacionados con la intensificación y domesticación de camélidos, como así también variabilidad en otros aspectos como la tecnología (Yacobaccio 2001). El caso de APC aporta nueva información para la discusión de esta problemática. En este sentido, la alta representación de camélidos en los conjuntos de APC es coherente con la información proveniente de otros contextos para esta cronología. Por ejemplo, Yacobaccio (2001) destacó el aumento de los camélidos en los conjuntos puneños hacia finales del Holoceno medio.

Asimismo, la osteometría permitió detectar variabilidad en el tamaño de los camélidos de APC. Si bien la muestra es reducida, el hallazgo de un espécimen de tamaño bastante mayor al del guanaco actual e incluso superior a algunos estándares de llamas actuales, indicaría la presencia de un camélido domesticado y/o en proceso de domesticación. Esto coincide con valores osteométricos registrados en otros sitios de la Puna de Salta como Alero Cuevas, que hacia esta cronología también evidencia cambios relacionados con la domesticación y/o introducción de camélidos domesticados (López 2008). A su vez, estos cambios son concordantes con la evidencia osteométrica de la capa de APC fechada en 3884 años AP, dónde se registran tamaños asignables a camélidos domesticados (López 2013; López y Restifo 2012).

Por otra parte, hasta el momento no se detectaron cambios tecnológicos notables en esta capa. Al contrario de APC, la tecnología de hojas prolifera en el sitio Alero Cuevas como forma base de los artefactos lanceolados unifaciales o Saladillo, comunes para esta cronología en algunos sectores de la Puna de Salta y Jujuy (Fernández 1983; Hoguin 2014; López y Restifo 2012). Algunos especímenes de estas características fueron hallados en superficie en la cuenca de Pocitos, aunque no se 
registraron en capa por el momento.

Como se ha señalado en otros trabajos (López 2013; López et al. 2013), APC y la cuenca de Pocitos en general, parecen mostrar una alta variabilidad de morfologías de puntas de proyectil y materias primas para contextos del Holoceno medio y comienzos del Holoceno tardío. Esto podría ser producto de los procesos de circulación de personas, recursos e información necesarios para la adaptación en ambientes ecológicamente desfavorables como la cuenca de Pocitos. En este sentido, las morfologías de las puntas de proyectil son comparables con otras registradas en distintas áreas de la Puna como Antofagasta de la Sierra en Catamarca o Susques en Jujuy (Hocsman 2006; Hoguin 2014). Por su parte, las materias primas tales como las obsidianas y las andesitas provienen de distintas fuentes. Este es el caso de obsidianas de Archibarca o Zapaleri, ambas localizadas a más de $100 \mathrm{Km}$ de APC. Esto refuerza la idea de Pocitos como un espacio en el que circularon recursos provenientes desde distintos espacios de la Puna, tanto hacia el sur como hacia el norte.

Para concluir, se plantea que los análisis realizados sobre el conjunto de APC aportan nueva información para el estudio de los procesos de cambio hacia finales del Holoceno medio. En futuras campañas, se profundizará la excavación del abrigo con el objetivo de ampliar las muestras y avanzar en la problemática de este trabajo.

\section{Bibliografía}

Aldenderfer, M. 1998. Montane Foragers, Asana and the south-central Andean foragers. University of lowa press. lowa.

Aschero, C. 1983. Ensayo para una clasificación morfológica de artefactos líticos aplicada a estudios tipológicos comparativos. Informe presentado al Consejo Nacional de Investigaciones Científicas y Técnicas (CONICET). Buenos Aires.

Aschero, C. 1994. Reflexiones desde el Arcaico Tardío (6000-3000 AP). Rumitacana. Revista de Antropología 1 (1): 13-17.

Cartajena, I, Núñez, L. y M. Grosjean. 2007. Camelid domestication on the western slope of the Puna de Atacama, northern Chile. Anthropozoologica 42 (2): 155-173.

Fernández, J. 1983. Río Grande. Exploración de un centro precerámico en las altas montañas de Jujuy, Argentina. Ampurias 45/46: 54-83.

Hocsman, S. 2006. Producción lítica, variabilidad y cambio en Antofagasta de la Sierra ca. 5500-1500 AP. Tesis de doctoral. Facultad de Ciencias naturales y Museo, La Plata. Inédita.
Hoguin, R. 2014. Secuencia cronológica y tecnología lítica en la Puna Seca y Salada de los Andes Centro-Sur para el Holoceno temprano y medio a través del ejemplo de Susques. Relaciones de la Sociedad Argentina de Antropología 39-2: 333-364.

Jones, G. y C. Beck. 1992. Chronological Resolution in Distributional Archaeology. J. Rossignol \& L. Wandnide ed., Space, Time and Archaeological Landcapes, 167192, Interdisciplinary Contributions to Archaeology, Plenum Press, New York \& London.

López, G. 2008. Arqueología de Cazadores y Pastores en Tierras Altas: Ocupaciones humanas a lo largo del Holoceno en Pastos Grandes, Puna de Salta, Argentina. BAR S1854, South American Archaeology Series 4. Oxford.

López, G. 2013. Ocupaciones humanas y cambio a lo largo del Holoceno en abrigos rocosos de la Puna de Salta, Argentina: Una perspectiva regional. Chungara, Revista de Antropología Chilena 45 (3): 411-426.

López, G. y F. Restifo. 2012. The Middle Holocene intensification and domestication of camelids in north Argentina, tracked by zooarchaeology and lithics. Antiquity 86: 1041-1054.

López, G. y F. Restifo. 2014. Procesos de diversificación, intensificación y domesticación durante el Holoceno en las tierras altas del norte de Argentina y Chile: aportes desde la Puna de Salta. Comechingonia 18 (2): 95 - 116.

López, G., F. Coloca y J. Orsi. 2013. Human Occupations during the Middle Holocene and Beginning of the Late Holocene the Pocitos Basin, Puna of Salta, Argentina: New Research and Regional Comparison. Quaternary International 307: 50-57.

Meadow, R. 1987. Techniques for comparing bone measurement data from small samples. Trabajo presentado en el Northeastern Faunal Analysis Conference. Connecticut.

Mengoni Goñalons, G. y H. Yacobaccio. 2006. The Domestication of South American Camelids. A View from the South-Central Andes. Editado por M. Zeder, D. Bradley, E. Emshwiller y B Smith, Documenting domestication, New genetics and archaeological paradigms, 228-243, University of California Press, Berkeley.

Muscio, H. 2012. Modelling demographic dynamics and cultural evolution: The case of the early and midHolocene archaeology in the highlands of South America. Quaternary International 256, 19-26.

Núñez, L., M. Grosjean y I. Cartajena. 2005. Ocupaciones humanas y paleoambientes en la Puna de Atacama. 
Catholic North University: Taraxacum, San Pedro de Atacama.

Olivera, D. 1997. La importancia del recurso camelidae en la Puna de Atacama entre los 10000 y los 500 años AP. Estudios Atacameños 14: $29-41$.
Yacobaccio, H. 2001. Cazadores complejos y domesticación de camélidos. Editado por G. Mengoni Goñalons, D. Olivera y H. Yacobaccio, El Uso de los Camélidos A Través del Tiempo, 261-281, Ediciones del tridente, Buenos Aires. 